\title{
A simple method for fabricating phase-shifted fibre Bragg gratings with flexible choice of centre wavelength
}

\author{
Edmon Chehura, Stephen W. James, and Ralph P. Tatam* \\ Engineering Photonics Group, School of Engineering, \\ Cranfield University, Cranfield, Bedford, MK43 0AL, UK.
}

\begin{abstract}
A simple technique for fabricating phase-shifted fibre Bragg gratings (PSFBGs) without the use of a phase-shifted phase mask is presented. Two, 3-mm long, standard fibre Bragg gratings (FBGs) were inscribed sequentially in singlemode fibre at the same Bragg wavelength such that the FBGs physically overlapped by one grating period. This induces a spectral-hole in the middle of the reflection spectrum of a standard FBG, equivalent to a $\pi$-phase shifted FBG. The flexibility of the technique in writing PSFBGs at any choice of wavelength is demonstrated. The results show that PSFBG devices produced by this method are highly reproducible and the process is fast.
\end{abstract}

Keywords: Fibre Bragg grating, FBG, phase shift, pi, $\pi$, PSFBG, sensors

\section{INTRODUCTION}

The transmission spectrum of a phase-shifted fibre Bragg grating (PSFBG) is characterised by a narrow bandpass that is usually of the order of a few tens of picometres. These fibre devices have found wide use in distributed feedback (DFB) fibre lasers, providing very stable narrow linewidth wavelength sources ${ }^{1}$. PSFBGs are increasingly being employed as high finesse transmission filters ${ }^{2}$, switching elements in dense wavelength division multiplexing (WDM) optical communication systems ${ }^{3}$, and in sensors for the measurement of a wide range of parameters ${ }^{4}$.

A number of fabrication techniques for PSFBGs have been investigated all of which exhibit limitations. The most reliable technique that produces high quality PSFBGs involves the use of a phase-shifted phase mask ${ }^{5}$ which inscribes the grating by a single exposure process. This method is limited to writing gratings at a single wavelength per phase mask and the phase-shifted phase mask is relatively expensive. PSFBGs have also been fabricated using the Moiré method $^{6}$ in a double exposure process. Two standard FBGs are spatially co-located and are sequentially written with the fabrication conditions altered between exposures such that the Bragg wavelengths of the FBGs' central wavelengths differ slightly (e.g. by $0.3 \mathrm{~nm}$ ). Methods that have been used to induce the difference in the Bragg wavelengths include slight tuning of the emission wavelength of the writing laser ${ }^{7}$, spatial displacement of the fibre perpendicular to the fibre axis (i.e. either towards or away from the focussing lens) ${ }^{6}$, and the application of strain to the fibre ${ }^{8}$ after writing the first FBG. High quality PSFBGs have been achieved with the Moire fabrication method but the method requires high precision if the correct phase shift is to be induced. A technique known as UV post-processing ${ }^{9}$ has also been investigated in PSFBG fabrication. The method entails raising the local refractive index of part of an FBG by focussing a UV beam to a small spot thereby creating a phase shift within the FBG structure. The method requires accurate control of the beam spot-size and positioning in the FBG if good quality PSFBGs are to be achieved and also the refractive index trimming process can be time consuming. Several other variations to the techniques discussed above have been demonstrated ${ }^{10,11}$ but all exhibit similar limitations.

This paper describes a simple technique for fabricating high quality PSFBGs at any required centre wavelengths using the standard FBG side writing techniques ${ }^{12,13}$ in a sequential double UV exposure process. Two standard FBGs are written at the same Bragg wavelength such that they have a spatial overlap equivalent to a single grating period. This procedure raises the local refractive index of the central intersection of the two FBGs, leading to the formation of a phase shift. The fabrication process is fast, repeatable, has flexible choice in Bragg wavelength and fabricates high quality PSFBGs.

\footnotetext{
*r.p.tatam@cranfield.ac.uk, phone 441234 754630; fax 441234 752452; http://www.cranfield.ac.uk/soe/departments/processsystems/engineeringphotonics
} 


\section{EXPERIMENTAL DETAILS}

All standard FBG fabrication systems ${ }^{12-14}$ have been used to write the PSFBGs reported in this work and figure 1a describes one of the configurations. The phase mask is used as a beam splitter and mirrors 1 and 2 select the \pm 1 diffraction orders of the UV laser beam, and redirect them to interfere at the fibre. The mirrors are mounted on rotary stages that are computer controlled to change the angle $\theta_{c}$, allowing FBGs of different centre wavelengths to be fabricated. A single cylindrical lens is used to focus the two beams so that they interfere at the fibre. The fibre is clamped onto a translation stage (PI, M-150.11) that is controlled by the computer through an RS232 cable. The translation stage has a maximum travel range of $50 \mathrm{~mm}$, resolution of $8 \mathrm{~nm}$ and is backlash free. A Michelson interferometer was configured alongside the FBG fabrication experiment to perform an independent measurement of the translation distance of the stage by counting the passage of interference fringes. The interferometer consists of a single frequency $\mathrm{HeNe}$ laser, mirrors M3 and M4, beam splitter and a detector D2. M4 is attached to the translation stage.

The FBG devices were fabricated using $30 \mathrm{~mW}$ of power from a $25 \mathrm{~Hz}$ pulsed Nd:YAG pumped dye laser ${ }^{13}$ operating at $248 \mathrm{~nm}$. The FBG was interrogated using a tuneable external cavity laser source (TUNICS-Plus CL) with $1 \mathrm{pm}$ resolution and a scan rate of $100 \mathrm{~nm} / \mathrm{s}$. An FBG was written into a singlemode photosensitive fibre (Fibercore, PS1250) at a specific centre wavelength until the required reflectivity was achieved. Next, the writing laser beam was blocked and the stage was translated under computer control through a distance equal to the length of the FBG less one grating period (figure $1 \mathrm{~b}$ ). Diagram B in Figure $1 \mathrm{~b}$ shows the fibre that was translated after writing $\mathrm{FBG}_{1}$ in order to prepare it for the fabrication of the second FBG. $\mathrm{FBG}_{2}$ was fabricated at the new fibre position, without changing the fabrication configuration, until the spectral-hole that develops in the middle of the FBG reflection spectrum had grown to its maximum depth and the amplitudes of the two peaks either side of it had become approximately equal. The minimum fabrication time required for $\mathrm{FBG}_{2}$, leading to a fully grown spectral-hole, is equivalent to the time taken to write $\mathrm{FBG}_{1}$. If the fabrication time for $\mathrm{FBG}_{2}$ is made longer than that of $\mathrm{FBG}_{1}$, the reflectivity of the PSFBG will increase. This provides a means to control the final reflectivity of the PSFBG device, if required. The phase-shifted FBG produced by this procedure is approximately twice the length of the individual FBGs as the spatial overlap between the FBGs is only one grating period. Diagram $\mathrm{C}$ in Figure $1 \mathrm{~b}$ illustrates the raised refractive index modulation in the intersection of the two FBGs, which leads to a phase shift.

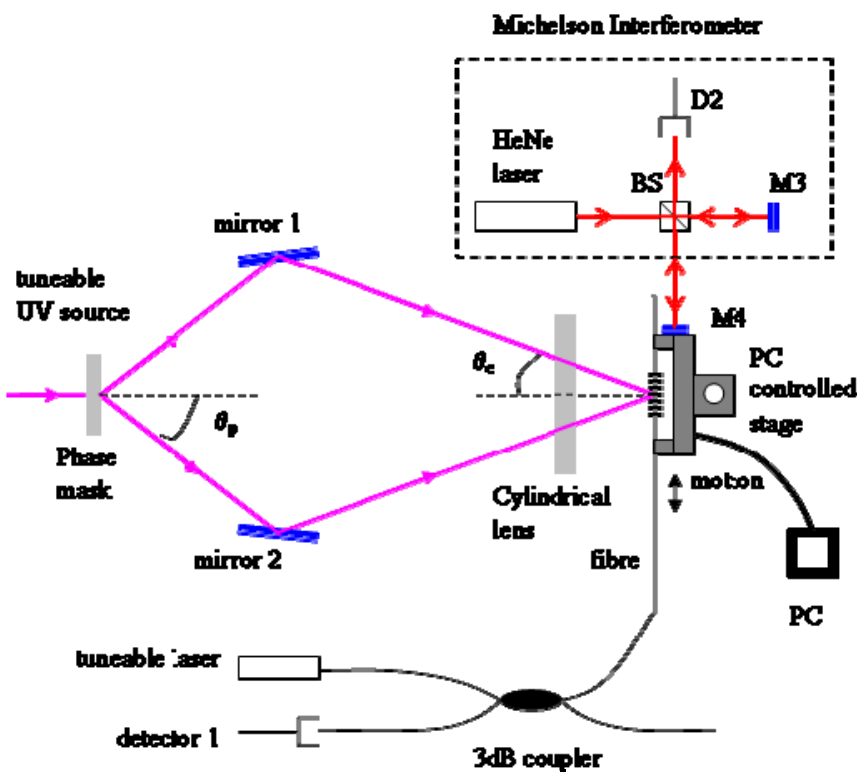

(a)

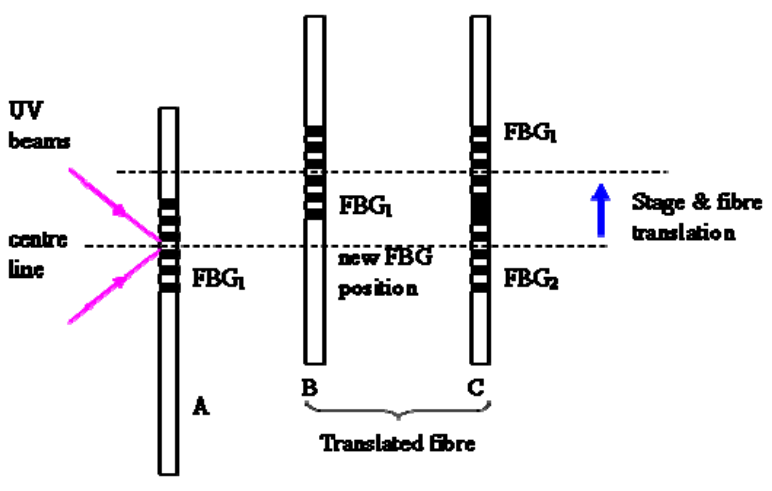

(b)

Figure 1: (a) A standard FBG fabrication (Interferometer) system ${ }^{13}$ adapted to fabricating phase-shifted FBGs and (b) shows fibre translation at FBG writing conditions. M; mirrors, D2; detector, PC; computer, and BS; beam splitter. 


\section{RESULTS AND DISCUSSION}

The blue curve in Figure 2a shows the reflection spectrum of a 3-mm long FBG that was written into a singlemode photosensitive fibre (Fibercore, PS1250) at a centre wavelength of about $1533.6 \mathrm{~nm}$ achieving a reflectivity of $30 \%$. When the writing laser beam was blocked the stage was translated by computer control through a distance of $3 \mathrm{~mm}$ less one grating period $( \pm 8 \mathrm{~nm}$ ) (figure $1 \mathrm{~b}$ ) after which a second FBG of the same centre wavelength was inscribed at the new fibre position using the same fabrication conditions as before. The red curve in Figure 2a shows the reflection spectrum produced by the formation of the phase-shifted FBG. A spectral-hole develops in the middle of the reflection spectrum of the first FBG (Figure 2a). The fabrication process is halted after the spectral-hole has developed to its maximum depth and after the peaks on either side have attained approximately equal amplitudes. The phase-shifted FBG is approximately twice the length of the individual FBGs (i.e. $6 \mathrm{~mm}$ ) and its reflectivity is about $60 \%$. Figure $2 \mathrm{~b}$ shows the reflection spectrum of a second phase-shifted FBG that was fabricated at the same centre wavelength of $1533.6 \mathrm{~nm}$ using the same fabrication conditions as the first PSFBG. The comparison of the spectra in Figure $2 \mathrm{a}$ and $2 \mathrm{~b}$ shows that the fabrication technique presented here is repeatable, providing reproducible spectral characteristics.

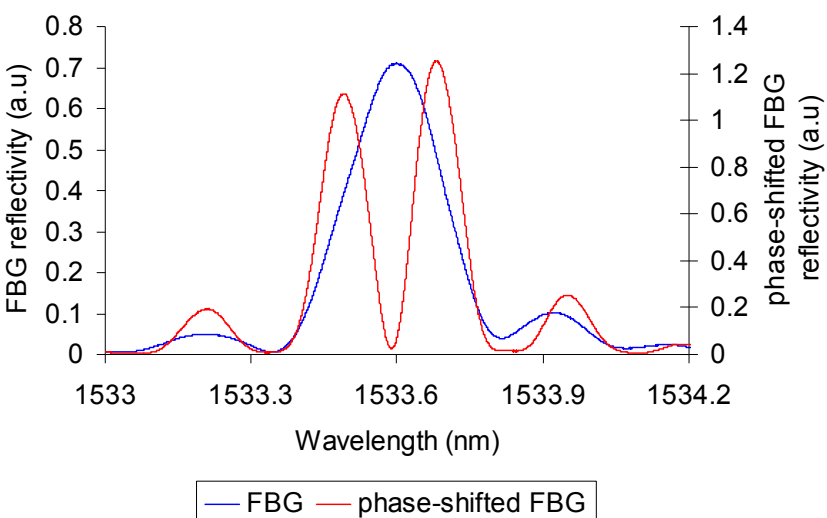

(a)

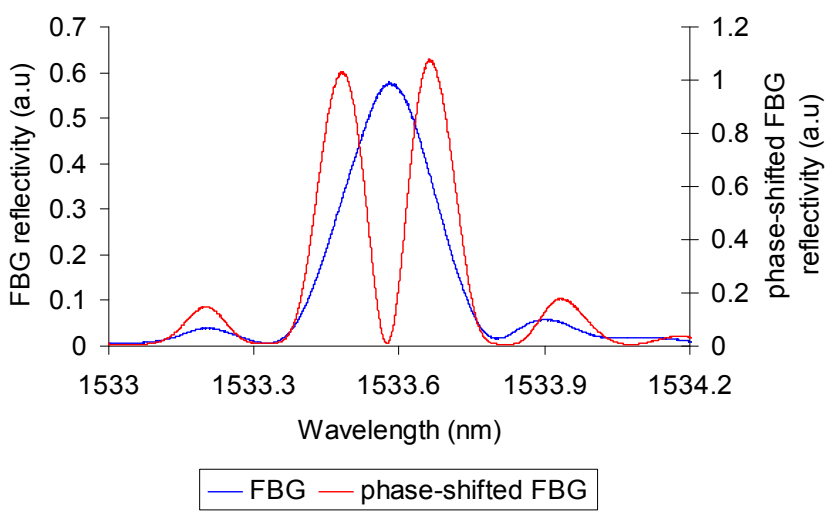

(b)

Figure 2: Reflection spectra of phase-shifted FBGs (red line) fabricated by partially overlapping 2 FBGs. The blue line is the spectrum of the $1^{s t} F B G$ written before the stage is translated. Plot (b) is a repeat fabrication of $(a)$.

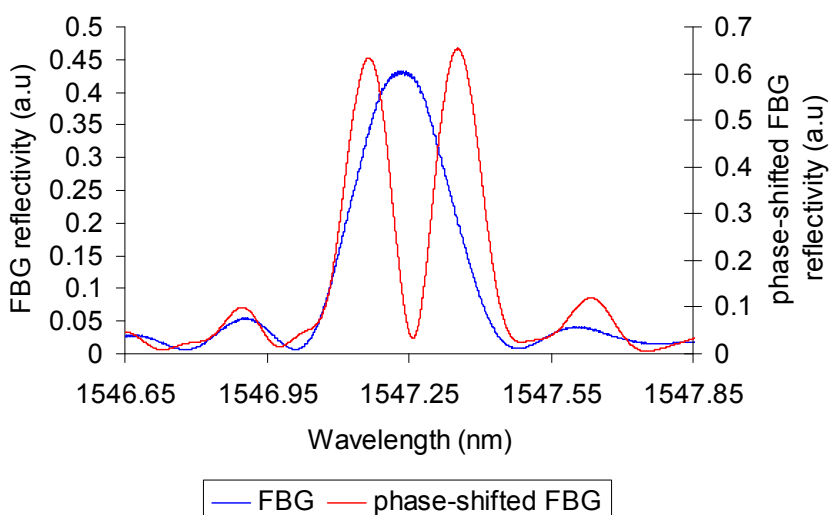

(a)

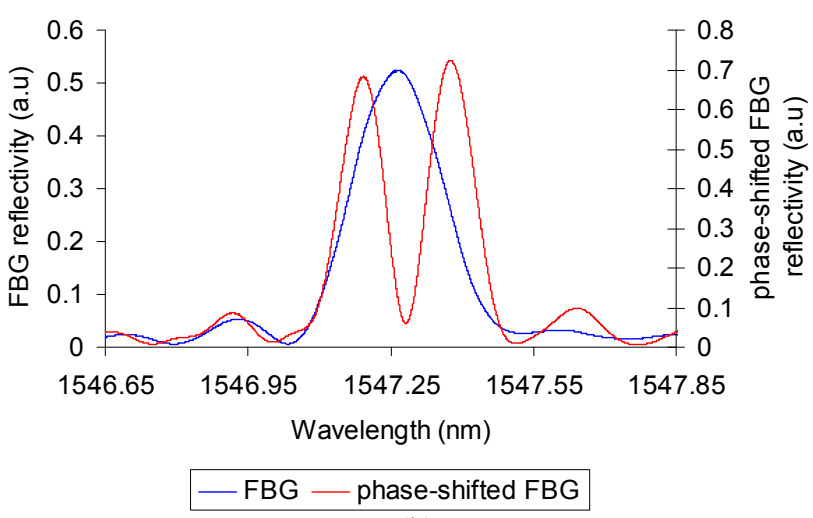

(b)

Figure 3: Reflection spectra of phase-shifted FBGs (red line) fabricated by partially overlapping 2 FBGs. The blue line is the spectrum of the $1^{s t} F B G$ written before the stage is translated. Plot (b) is a repeat fabrication of $(a)$.

Angle $\theta_{c}$ in Figure 1 was readjusted in order to write phase-shifted FBGs at a different centre wavelength. A 3-mm long standard FBG of up to $20 \%$ reflectivity was fabricated at a centre wavelength of $1547.25 \mathrm{~nm}$. The reflection spectrum of this FBG is denoted by the blue curve in Figure 3a. A second 3-mm long standard FBG was written at a new fibre position after the stage was translated by $3 \mathrm{~mm}$ less one grating period. The FBG was written using the same fabrication conditions as for the first FBG. A phase-shifted FBG is produced because the two FBGs are partially overlapping (by 
one grating period) thus raising the refractive index in the region of overlap of the gratings. The reflection spectrum of the phase-shifted FBG (30\% reflectivity) that was produced in this way is denoted by the red curve in Figure $3 \mathrm{a}$. The fabrication process was halted when the spectral-hole that developed in the centre of the standard FBG spectrum had grown to a maximum depth and when the two side peaks were approximately equal in amplitude (Figure $3 \mathrm{a}$ ). Figure $3 \mathrm{~b}$ shows the spectrum of a second phase-shifted FBG that was fabricated without changing the writing configuration. The fabrication technique presented here offers very good repeatability as demonstrated by the two phase-shifted FBGs of Figures $3 \mathrm{a}$ and $3 \mathrm{~b}$ that exhibit reproducible spectral characteristics. Figure 2 and Figure 3 also demonstrate the flexibility of this fabrication technique in fabrication of wavelength-multiplexed phase-shifted FBGs. The linewidth exhibited by the spectral-hole was $\sim 82 \mathrm{pm}$ at full width and half maximum (FWHM). The linewidth compares very well with the linewidths of PSFBGs fabricated using the Moire fabrication technique ${ }^{6-8}$. Linewidths of PSFBGs fabricated using phase-shifted phase masks can be as narrow as $10 \mathrm{pm}^{5}$.

\section{CONCLUSION}

A simple, versatile, cost-effective and fast method for fabricating efficient phase-shifted FBGs has been presented. High quality phase-shifted FBGs with reproducible spectral characteristics have been achieved, demonstrating the high repeatability offered by this fabrication technique when fabricating phase-shifted FBG devices at the same centre wavelengths such that they all exhibit same spectral characteristics. Phase-shifted FBGs were also fabricated at very different centre wavelengths, demonstrating the flexibility of the technique in fabricating wavelength-multiplexed phaseshifted FBG devices in a fast and cost-effective way.

\section{ACKNOWLEDGEMENTS}

This work was supported by the Engineering and Physical Sciences Research Council (UK).

\section{REFERENCES}

1. J. T. Kringlebotn, J. L. Archambault, L. Reekie, and D. N. Payne, " $\mathrm{Er}^{3+}: \mathrm{Yb}^{3+}$-codoped fiber distributedfeedback laser", Opt. Lett., 19, pp. 2101-2103, 1994.

2. I. C. M. Littler, M. Rochette, and B. J. Eggleton, "Adjustable bandwidth dispersionless bandpass FBG optical filter", Opt. Express, 13, pp. 3397-3407, 2005.

3. G. P. Agrawal, and S. Radic, "Phase-shifted fiber Bragg gratings and their applications for wavelength demultiplexing", IEEE Photon. Technol. Lett., 6, pp. 995-997, 1994.

4. M. LeBlanc, S. T. Vohra, T. E. Tsai, and E. J. Friebele, "Transverse load sensing by use of pi-phase-shifted fiber Bragg gratings", Opt. Lett., 24, pp. 1091-1093, 1999.

5. R. Kashyap, P. F. Mckee, and D. Armes, "UV written reflection grating structures in photosensitive optical fibres using phase-shifted phase masks", Electron. Lett., 30, pp. 1977-1978, 1994.

6. D. C. J. Reid, C. M. Ragdale, I. Bennion, D. J. Robbins, J. Buus, and W. J. Stewart, "Phase-shifted Moiré grating fibre resonators", Electron. Lett., 26, pp. 10-12, 1990.

7. S. Legoubin, E. Fertein, M. Douay, P. Bernage, P. Niay, F. Bayon, and T. Georges, "Formation of Moiré grating in core of germanosilicate fibre by transverse holographic double exposure method", Electron. Lett., 27, pp. 1945-1947, 1991.

8. L. Zhao, L. Li, A. Luo, J. Z. Xia, R. H. Qu, and Z. Fang, "Bandwidth controllable transmission filter based on Moiré fiber Bragg grating", Optik, 113, pp. 464-468, 2002.

9. J. Canning, and M. G. Sceats, " $\pi$-phase-shifted periodic distributed structures in optical fibres by UV postprocessing”, Electron. Lett., 30, pp. 1344-1345, 1994.

10. L. Xia, P. Shum, and C. Lu, "Phase-shifted bandpass filter fabrication through CO2 laser irradiation", Opt. Express, 13, pp. 5878-5882, 2005.

11. L. Wang, B. Chen, J. Chen, L. Chang, G. Li, A. Sun, and Z. Lin, "A simple method to fabricate phase-shifted fiber grating", Chinese Opt. Lett., 5, pp. S82-S83, 2007.

12. K. O. Hill, B. Malo, F. Bilodeau, D. C. Johnson, and J. Albert, "Bragg gratings fabricated in monomode photosensitive optical fibre by UV exposure through a phase mask", Appl. Phys. Lett., 62, pp. 1035-1037, 1993.

13. G. Meltz, W. W. Morey, and W. H. Glenn, "Formation of Bragg gratings in optical fibres by a transverse holographic method", Opt. Lett., 14, pp.823-825, 1989.

14. M. L. Dockney, S. W. James, and R. P. Tatam, "Fibre Bragg gratings fabricated using a wavelength tuneable laser source and a phase mask based interferometer", Meas. Sci. Technol., 7, pp. 445-448, 1996. 